Optically induced electrokinetic concentration and sorting of colloids

This article has been downloaded from IOPscience. Please scroll down to see the full text article.

2010 J. Micromech. Microeng. 20015022

(http://iopscience.iop.org/0960-1317/20/1/015022)

View the table of contents for this issue, or go to the journal homepage for more

Download details:

IP Address: 152.78.66.11

The article was downloaded on 06/05/2010 at 10:22

Please note that terms and conditions apply. 


\title{
Optically induced electrokinetic concentration and sorting of colloids
}

\author{
Stuart J Williams ${ }^{1}$, Aloke Kumar ${ }^{2}$, Nicolas G Green ${ }^{3}$ and \\ Steven T Wereley ${ }^{2}$ \\ 1 Department of Mechanical Engineering, University of Louisville, Louisville, KY 40292, USA \\ 2 Birck Nanotechnology Center, Purdue University, West Lafayette, IN 47907-2057, USA \\ ${ }^{3}$ School of Electronics and Computer Science, University of Southampton, Southampton S017 113J, UK \\ E-mail: wereley@purdue.edu
}

Received 2 September 2009, in final form 5 October 2009

Published 2 December 2009

Online at stacks.iop.org/JMM/20/015022

\begin{abstract}
We demonstrate an optically induced ac electrokinetic technique that rapidly and continuously accumulates colloids on a parallel-plate electrode surface resulting in a crystalline-like aggregation. Electrothermal hydrodynamics produce a microfluidic vortex that carries suspended particles toward its center where they are trapped by local ac electrokinetic hydrodynamic forces. We characterize the rate of particle aggregation as a function of the applied ac voltage, ac frequency and illumination intensity. Hundreds of polystyrene particles $(1.0 \mu \mathrm{m})$ suspended in a low conductivity solution $\left(2.4 \mathrm{mS} \mathrm{m}^{-1}\right)$ were captured at a range of voltages $\left(5-20 \mathrm{~V}_{\mathrm{pp}}\right)$ and frequencies $(20-150 \mathrm{kHz})$ with an optical power of approximately $20 \mathrm{~mW}$. This technique was not restricted to near infrared $(1064 \mathrm{~nm})$ illumination and was also demonstrated at $532 \mathrm{~nm}$. The sorting capability of this technique was demonstrated with a solution containing $0.5 \mu \mathrm{m}, 1.0 \mu \mathrm{m}$ and $2.0 \mu \mathrm{m}$ polystyrene particles. This dynamic optically induced technique rapidly concentrates, sorts and translates colloidal aggregates with a simple parallel-plate electrode configuration and can be used for a variety of lab-on-a-chip applications.
\end{abstract}

M] This article features online multimedia enhancements

(Some figures in this article are in colour only in the electronic version)

\section{Introduction}

Recently, we introduced an ac electrokinetic technique that can rapidly accumulate, translate and pattern micro- and nanoparticles and concentrate them on the surface of an electrode [1-3]. Rapid electrokinetic patterning (REP) is optically induced, offering dynamic control of the aggregation. In brief, the applied illumination creates thermal gradients, which, in the presence of an ac electric field, induce a microfluidic vortex [4]. This vortex carries particles toward its center and, at low ac frequencies $(<150 \mathrm{kHz})$ and low conductivity media $\left(<10.0 \mathrm{mS} \mathrm{m}^{-1}\right)$, particles are concentrated on the electrode surface. This process is illustrated in figure 1. We have demonstrated dynamic particle manipulation with polystyrene, latex and silica colloids (49 $\mathrm{nm}$ to $3.0 \mu \mathrm{m}$ ) at applied ac signals $1-150 \mathrm{kHz}$ and $0-20$ volts peak-to-peak $\left(\mathrm{V}_{\mathrm{pp}}\right)$ with illuminations under $100 \mathrm{~mW}$. These colloidal accumulations can be readily concentrated, sorted, translated and patterned anywhere on the surface of the electrode substrate by dynamically changing either the geometry of the optical landscape or the ac signal.

Due to its ability to concentrate particles in a crystallinelike aggregation, REP can be applied to the assembly of colloids or biological cells to create artificial architectures including photonic crystals [5] or bioengineered tissues [6]. Since this technique simultaneously induces fluid motion to rapidly and continuously concentrate a sample, REP can be used to improve the efficiency of lab-on-a-chip sensors and processes, including bead-based assays [7]. In addition, REP colloidal accumulation is frequency dependent, enabling sorting and characterization of species based on their ac electrokinetic behavior.

REP overcomes some of the limitations of other noncontact particle-handling methods such as optical trapping [8-12], electrophoresis [13-15] and dielectrophoresis (DEP) [16-20]. Optical trapping suffers from limited throughput, 


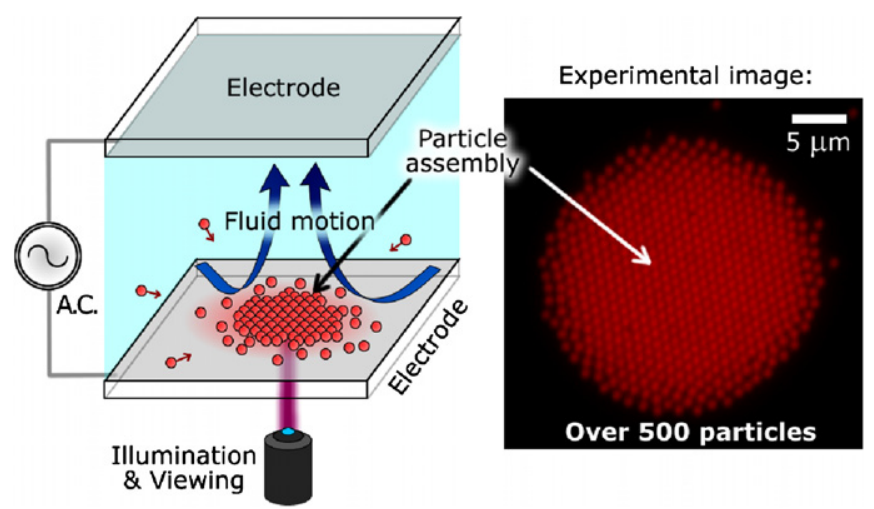

Figure 1. Illustration of the rapid electrokinetic patterning (REP) process. Optically induced electrokinetic mechanisms concentrate suspended particles in a crystalline-like monolayer. The experimental image shows a concentration of over $5001.0 \mu \mathrm{m}$ particles. Illustration modified from [2]

while electrokinetic techniques are limited in applications due to permanently patterned electrode features. REP overcomes these limitations by coupling optics with electrokinetics and carefully balancing the resulting forces.

Laser-induced electrokinetic fluid motion has been investigated previously [21]. The researchers used a focused YAG laser operating at $1064 \mathrm{~nm}$ and presented flow visualization results of a microvortex they termed an optoelectrostatic microvortex (OEMV). This phenomenon was observed with broad illumination [22] and fluid motion was attributed to optically induced electrothermal hydrodynamics [23]. This technique can be utilized for microfluidic pumping [24]; within REP the optically induced fluid motion is coupled with low-frequency colloidal electrokinetics to expedite particle concentration.

Arbitrary electrokinetic colloidal aggregation at low ac frequencies have also been explored [25-28]. Particle aggregates have been patterned previously using an electric field coupled with broad illumination geometries applied to a photosensitive platform [29]; however, this patterning process can take hours to complete. Illumination-induced thermophoresis and convection have been used to form twodimensional crystalline structures [30]. One limitation is that it is difficult to sort different particles during assembly.

Another separate, dynamic, optically induced electrokinetic technique is termed optoelectronic tweezers (OET) and can handle particles with induced dielectrophoresis $[19,20]$, ac electroosmosis [31, 32] or electrothermal hydrodynamics [33]. Both REP and OET are optically induced electrokinetic techniques that induce fluid motion and particle manipulation. The distinct difference between REP and OET is that the latter utilizes photoconductive materials such as amorphous silicon, whereas REP does not. OET utilizes photoconductive materials and applied illumination patterns controlled with a digital micromirror display (DMD) [19] or LCD [34] resulting in non-uniform electric fields to induce electrokinetic mechanisms. By comparison, REP occurs on the electrode surface of a parallel-plate environment (uniform electric field) with an applied highly focused beam of light. Without the need of photoconductive materials, REP chips are easier to fabricate and incorporate with existing lab-on-a-chip technologies. However, typical illumination intensities of REP $(<100 \mathrm{~mW})$ are greater than those used in OET $(<10 \mathrm{~mW})$. The physical mechanisms driving REP are discussed in the following section.

In this paper, we expand upon our initial disclosure of REP [1], explaining and confirming its electrokinetic mechanisms. Further, the rate of particle accumulation is characterized by ac voltage, ac frequency and illumination intensity. Last, the frequency-dependent behavior of REP was used to sort particles by size and by type based on their polarization behavior.

\section{Theory}

REP is a technique that couples illumination-induced electrothermal hydrodynamics with low-frequency colloidal ac electrokinetics to accumulate, sort and pattern particles. REP has been introduced previously [1], with the theories of the dominating physics explained in detail below.

\subsection{Overview of rapid electrokinetic patterning (REP)}

The REP technique is illustrated in figure 1. A low conductivity $\left(<10.0 \mathrm{mS} \mathrm{m}^{-1}\right)$ liquid sample containing colloids was introduced between two electrodes arranged in a parallel-plate configuration separated by $50 \mu \mathrm{m}$. An ac signal was applied between these electrodes at frequencies typically less than $150 \mathrm{kHz}$. A highly focused optical landscape was applied to the surface of either electrode to induce electrothermal fluid motion. A microfluidic vortex was created, with the center of the recirculation located at the laser focal point.

This microfluidic vortex carries suspended colloids toward the center of the optical illumination where they aggregate. The colloidal concentration is typically a crystalline-like aggregation. These particle assemblies can be patterned, taking the general shape of the illuminated regions. The location and geometry of optical landscapes are computercontrolled with spatial light modulators (SLMs). For experiments herein, the applied optical landscape was a simple focused beam of light, similar in nature to optical tweezers. Even though optical trapping systems that incorporate SLMs can be expensive, low-cost optical trapping systems could be used to induce REP [35] or other similar systems with a lower laser power source.

With REP, particle assemblies can be readily translated on the electrode surface (supplementary video 1; available online at stacks.iop.org/JMM/20/015022/mmedia). REP can occur on either electrode surface within the parallel-plate configuration. The electrode closest to the illumination source was indium tin oxide (ITO), a transparent conductor, deposited on glass. This allows for the illumination to pass through and REP to occur on either the top or bottom electrode surface.

\subsection{Overview of the physical mechanisms driving REP}

There is a combination of three mechanisms that are responsible for the continuous concentration of colloids in 


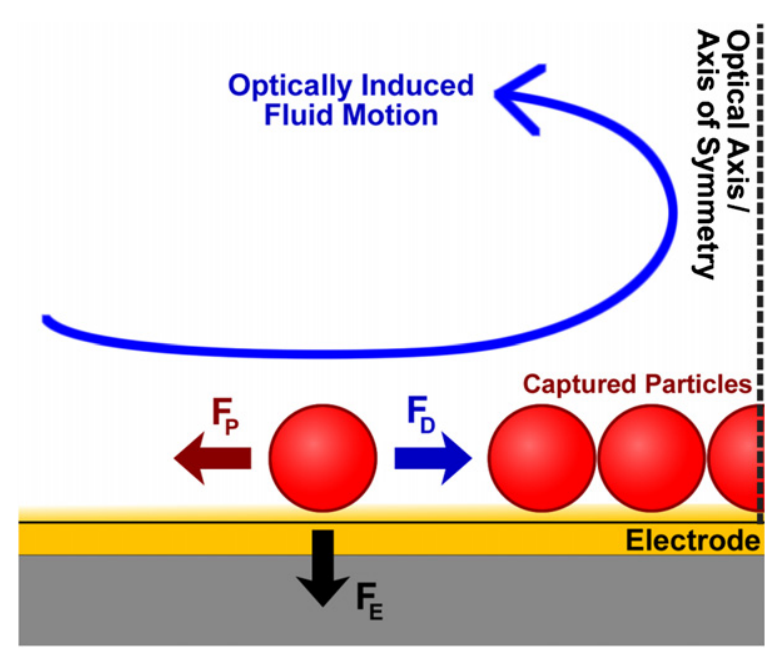

Figure 2. Illustration of the physical forces exerted on a single particle being captured with REP.

REP; forces are labeled on a single particle illustrated in figure 2. First, a particle is carried into the trapping region with optically induced fluid motion. The recirculating microfluidic vortex exerts a fluid drag on this particle, resulting in compact colloidal aggregation $\left(F_{D}\right.$ in figure 2$)$. Secondly, as the particle is carried toward the surface of the planar electrode, low-frequency electrokinetic forces trap the particle close to the electrode surface ( $F_{E}$ in figure 2$)$. Finally, the particles themselves exhibit particle-particle interactions due to a variety of electrokinetic mechanisms. Figure 2 illustrates these particle-induced interactions as a repulsive force $\left(F_{P}\right)$. Each of these three forces is discussed in more detail in the following sections.

\subsection{Electrothermal fluid motion in ac fields}

Thermal gradients within the fluid generate localized changes in fluid permittivity and conductivity. The electric field will act upon these dielectric gradients resulting in fluid motion [36]. The time averaged body force $\left(f_{e}\right)$ acting upon the fluid is given by [23]

$\left\langle f_{e}\right\rangle=\frac{1}{2} \operatorname{Re}\left[\frac{\sigma \varepsilon(\alpha-\beta)}{\sigma+\mathrm{i} \omega \varepsilon}(\nabla T \bullet \mathbf{E}) \mathbf{E}^{*}-\frac{1}{2} \varepsilon \alpha|\mathbf{E}|^{2} \nabla T\right]$

where Re refers the real part of the expression, $\mathbf{E}$ is the electric field, $\mathbf{E}^{*}$ is its complex conjugate, $T$ is the temperature, $\omega$ is the applied angular frequency, $\sigma$ and $\varepsilon$ are the conductivity and permittivity of the fluid, respectively, $\beta$ is $(1 / \sigma)(\partial \sigma / \partial T)$ and $\alpha$ is $(1 / \varepsilon)(\partial \varepsilon / \partial T)$.

The first term in equation (1) is the Coulomb force and the second term is the dielectric force; the former will dominate as the applied ac frequency decreases. Each expression is dependent on local temperature gradients $(\nabla T)$. Thermal gradients can be generated by Joule heating [23] or by illumination [21, 22, 24]. However, due to the uniform electric field within our current parallel-plate electrode configuration, the dominant source of non-uniform heat for our REP experiments comes from the highly focused optical illumination. This heating is highly non-uniform due to the highly focused light patterns that vary radically in intensity over a few micrometers. The induced fluid velocity depends on the illumination intensity (thermal gradient), the dielectric properties of the fluid and the applied ac signal (voltage and frequency). From equation (1), the fluid velocity $(v)$ is proportional to the square of the electric field $\left(E^{2}\right)$. Assuming Stokes' drag, the lateral force exerted on a particle $\left(F_{D}\right.$ figure 2) is proportional to fluid velocity and particle radius (a). Other factors such as hydrodynamic lifting forces (scaled as $v^{2}$ ) [37] and a particle's proximity to neighboring particles would influence these forces exerted on the particle. However, in REP the electrokinetic forces exerted on particles overcome hydrodynamic lifting forces and traps particles on the electrode surface- these electrokinetic forces are discussed next.

\subsection{Electrokinetic forces acting on colloids near the electrode surface}

Fagan et al [38-40] have extensively investigated the vertical motion and forces exerted on a single colloidal particle on the surface of an electrode when subjected to a uniform ac electric field. They observed the behavior of the colloid in order to understand the mechanisms responsible for its motion. Investigators extending back to Yeh et al [27] have theorized that localized electroosmotic flow was responsible for particle forces. Electrohydrodynamic flow is induced due to the interaction of the applied electric field with the polarization of the ionic double layer of the electrode and particle.

Ristenpart et al [28] predicted that these electrohydrodynamic forces scale with the square of the electric field strength $\left(E^{2}\right)$ and the inverse of frequency $\left(\omega^{-1}\right)$. This relationship provides a good estimation of particle forces for applied ac frequencies greater than $500 \mathrm{~Hz}$ [39]. The REP experiments herein were conducted with frequencies greater than $10 \mathrm{kHz}$ and therefore we can assume the above relationship for particle-electrode electrokinetic forces ( $F_{E}$ figure 2$)$.

The electrohydrodynamic forces occur in part due to the interaction of the electric field with the polarization of the particle's double layer. This polarization is attributed to the changes in surface charge density of the electrical double layer of the particle. Previous experiments have indicated that the dispersion of this polarization mechanism occurs at frequencies lower than the Maxwell-Wagner interfacial polarization [41-43]. This dispersion occurs at frequencies proportional to the particle's surface area $\left(a^{2}\right)$ [43]. At frequencies above this dispersion, the electrohydrodynamic holding forces become negligible and particles are released from the electrode surface and are carried away by fluid motion. REP can therefore sort different particles at various applied frequencies based on the dispersion of their polarized double layer.

It has also been observed that particles on the electrode surface are suspended with a height on the order of $100 \mathrm{~nm}$ [38-40]. This gap is the result of a balance of several forces including van der Waals attraction, electrostatic repulsion, electrohydrodynamic effects and gravity. The height of REP 
captured particles above the electrode was not able to be measured for our experiments, but this behavior may account for the observed confluent translation of particle aggregations across the electrode surface as the location of the focused illumination was altered (supplementary video 1; available online at stacks.iop.org/JMM/20/015022/mmedia).

\subsection{Electrokinetic particle-particle interactions}

The electrokinetic lateral motion of particles on the surface of an electrode has also been extensively investigated [28, 44-47]. The forces between particles $\left(F_{P}\right.$ figure 2$)$ can either be attractive or repulsive, and this behavior is dependent on a number of variables. Particle-particle interactions for frequencies greater than $500 \mathrm{~Hz}$ are a combination of electrohydrodynamic effects and dipole-dipole repulsive forces [28, 46].

Particle-particle and particle-electrode electrokinetic mechanisms are complex and are not completely understood. These electrokinetic phenomena are the subject of continued experimentation. These mechanisms are a function of ac frequency, electric field magnitude, particle size, electrolyte conductivity and electrolyte type. Therefore, the nature of REP should behave differently for a variety of conditions, and these changes will be a focus of future investigations in order to fully characterize REP. The results herein provide a foundation for future investigations as well as provide instruction necessary for incorporation and application of REP.

\section{Experimental details}

\subsection{Chip design and fabrication}

The experimental chip consists of microfluidic chambers sandwiched between two parallel-plate electrodes (figure 1). ITO-coated glass cover slips (170 $\mu \mathrm{m}$ thick) were used for the bottom electrode, while the top electrode was a second ITOcoated glass substrate for improved visualization. The height of the chamber was $50 \mu \mathrm{m}$ and constructed from thick doublesided adhesive tape with microfluidic features. The observed experimental area was placed approximately in the middle of the microfluidic chamber in order to avoid any influence of the wall on the local electric field. An ac signal was then applied between the parallel-plate electrodes, resulting in a uniform electric field in the experimental area.

\subsection{Illumination system and calibration}

An inverted Nikon TE2000U microscope equipped with a Nikon $40 \times$ water-immersion lens $(0.8$ N.A. and $2.0 \mathrm{~mm}$ working distance) was utilized for visualization. The illumination source for REP was a Nd:YAG laser-based holographic illumination system (Bioryx ${ }^{\circledR} 200$ from Arryx Inc., Chicago, USA) operating at $1064 \mathrm{~nm}$. Holographic optical landscapes were implemented by a computercontrolled spatial light modulator, allowing the user to easily repeat and reconfigure illumination patterns [11]. These strongly focused illumination patterns were used to drive the electrokinetic mechanisms behind REP. The laser power could be programmed to a maximum output of $2.0 \mathrm{~W}$; however, there was loss from the laser's delivery to the chip. The laser intensity was measured before the objective lens and calibrated for the following experiments - all stated experimental illumination intensities were with respect to these measured values.

\subsection{Particle preparation}

Carboxylate-modified fluorescent polystyrene particles (1.0 $\mu \mathrm{m}$, Invitrogen, OR, USA) were used to characterize our REP technique as a function of ac frequency, ac voltage and illumination intensity. The concentration of suspended colloids remained constant between experiments. This solution was prepared by diluting $20 \mu \mathrm{L}$ of a $2 \%$ solids particle solution with $2.0 \mathrm{~mL}$ of an aqueous solution of potassium chloride $(\mathrm{KCl})$ with a conductivity $(\sigma)$ of $2.4 \mathrm{mS} \mathrm{m} \mathrm{m}^{-1}$. For $1.0 \mu \mathrm{m}$ particles, this results in approximately 380 million particles per mL. Prepared samples were manually injected between the parallel-plate electrodes.

An additional sample with a mixture of three different particle types was prepared for frequency-dependent colloidal concentration experiments. This sample was prepared by adding $2 \%$ solids solution of $0.5 \mu \mathrm{m}(3 \mu \mathrm{L}), 1.0 \mu \mathrm{m}$ $(20 \mu \mathrm{L})$ and $2.0 \mu \mathrm{m}(100 \mu \mathrm{L})$ diameter carboxylate-modified polystyrene beads (Invitrogen, OR, USA) in a $2.0 \mathrm{~mL}$ aqueous $\mathrm{KCl}$ solution $\left(\sigma=2.4 \mathrm{mS} \mathrm{m}^{-1}\right)$.

Particles were soaked in the aqueous $\mathrm{KCl}$ solution for at least $30 \mathrm{~min}$ prior to testing to stabalize the particle zeta potential. A solution without particles was injected into the microchannel and left for at least $1 \mathrm{~h}$ to allow for the stabalization of the electrode surfaces [44]. REP experiments herein were repeated at least three times for validation of observed trends.

\subsection{Image acquisition and processing}

Images of the captured colloids were acquired with an interline transfer charge coupled device (CCD) camera (PCO 1600). When a series of images were acquired, the time interval between successive images was $1.0 \mathrm{~s}$ for the duration of the experiment ( $3 \mathrm{~min}$ ). Images were processed by a MATLAB program that determined the number of acquired particles. This program was modeled after an existing particle-locating algorithm [48] and modified to determine the location of particle centers with sub-pixel accuracy.

Between each experiment, the ac signal and illumination were deactivated and a fresh sample was injected. The previous colloidal aggregations were flushed to prevent interference with future trials.

\section{Results and discussion}

\subsection{Observation of colloidal particle accumulation in REP}

First, the electrokinetic mechanisms responsible for particle aggregation were investigated. Figure 3 shows a series of experimental images that were acquired when (a) both 

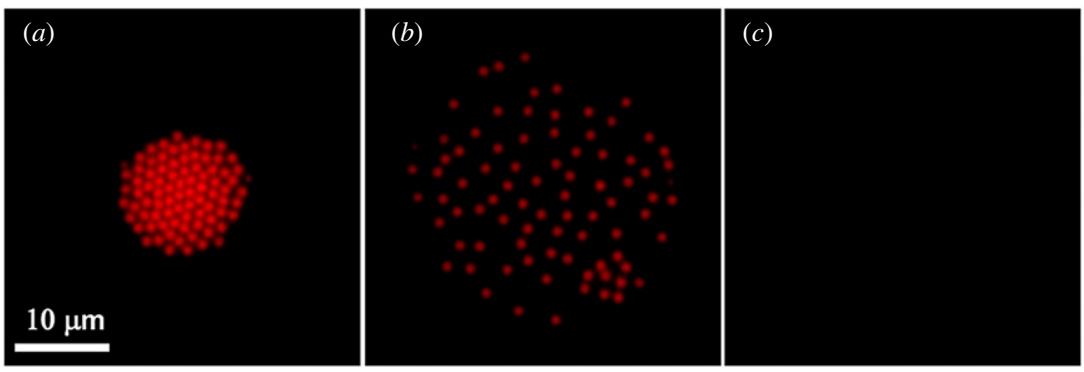

Figure 3. REP requires simultaneous application of illumination and electric fields. These images show $(a)$ both illumination and electric field applied, (b) electric field only and (c) illumination only.

illumination and the electric field were activated, (b) when the illumination was deactivated and (c) moments after the simultaneous reactivation of the illumination and deactivation of the electric field. First, $1.0 \mu \mathrm{m}$ polystyrene particles were concentrated at $18 \mathrm{~mW}, 100 \mathrm{kHz}$ and $13.5 \mathrm{~V}_{\mathrm{pp}}$ (figure $3(a)$ ). When the illumination was removed, the particles scattered but remained on the electrode surface (figure $3(b)$ ). For this scenario, there was no observed microfluidic vortex motion and therefore no fluid drag to compact the particle aggregation. Without fluid drag, the particles were repelled from each other due to induced dipole-dipole repulsive forces [28, 46]. This repulsion is the dominant force for our tested frequency range $(>10 \mathrm{kHz})$ and scales as the square of the applied field. These particles remain on the surface of the electrode due to particleelectrode hydrodynamic forces.

When the illumination was reapplied, the resulting lateral fluid drag overcame these particle-particle repulsive forces, concentrating the particles in the center again. However, when the electric field was turned off, the particles diffuse away into the bulk of the medium, away from the focal plane on the electrode surface (figure 3(c)). Without the applied electric field, the particles were not attracted to the electrode surface and the microfluidic vortex was not observed. In addition, it can be concluded that optical trapping forces were not responsible for particle accumulation. A video of this experiment is available in the online version of the journal at stacks.iop.org/JMM/20/015022/mmedia (supplementary video 2 ).

\subsection{Thermal and fluid mechanisms in REP}

Our previous investigations [1] reported REP with a parallelplate configuration where both electrodes were ITO on glass and our illumination wavelength was $1064 \mathrm{~nm}$. Further, it was reported that, for the same ITO/ITO electrode configuration, no accumulation was observed for an applied illumination wavelength of $532 \mathrm{~nm}$. This was due to the fact that the absorbance of water and ITO in the near-infrared is much greater in the visible spectrum [49-51] and, as a result, the thermal gradients necessary to drive REP are significantly greater with $1064 \mathrm{~nm}$ illumination.

Different wavelengths could be applied to a proper substrate material to induce REP. Additional experiments were conducted with one ITO electrode and one gold-coated electrode. Using $1064 \mathrm{~nm}$ illumination light, REP occurred on both electrode surfaces as expected. However, using $532 \mathrm{~nm}$ illumination with this ITO/Au chip resulted in REP on the gold surface and not on the ITO surface. Gold has a significant absorbance at $532 \mathrm{~nm}$ [49], and thermal gradients are produced from gold absorbing $532 \mathrm{~nm}$ light, as opposed to previous experiments where water and ITO were directly heated with $1064 \mathrm{~nm}$ illumination.

ITO itself absorbs infrared light, and previously lasers have been used to ablate and pattern ITO films [50, 51]. Ichikaway et al [52] locally heated islands of ITO films with $1064 \mathrm{~nm}$ illumination in order to increase the temperature of hydrogels by approximately $6{ }^{\circ} \mathrm{C}$ with an applied illumination intensity of $105 \mathrm{~mW}$. The heating of the ITO surface with the applied near-infrared illumination drives the microfluidic vortex. In addition, successful REP on the surface of a gold electrode with $1064 \mathrm{~nm}$ illumination is evidence that the illumination heats the liquid as well as the substrate in order to generate optically induced electrothermal fluid motion.

With this knowledge, REP is not restricted to a range of wavelengths and configurations can be designed which are illumination specific. For example, silver readily absorbs $300 \mathrm{~nm}$ light and silicon absorbs 600-1000 nm [49]. Therefore, REP can occur by heating either the fluid directly (water with infrared light), the electrode (gold with $532 \mathrm{~nm}$ or silver with $300 \mathrm{~nm}$ ) or the substrate (silicon with 600$1000 \mathrm{~nm})$.

The illumination-induced electrothermal microfluidic vortex has been investigated previously with highly focused $1064 \mathrm{~nm}$ light between parallel-plate electrodes [4]. In this configuration, an induced toroidal microfluidic vortex was observed in a plane normal to the electric field. Experiments showed that the velocity of the microvortex was proportional to voltage squared, which is consistent with equation (1). Further, the fluid velocity increased as frequency was decreased from 5.0 $\mathrm{MHz}$ down to $100 \mathrm{kHz}$. This frequency-dependent trend is expected from equation (1); for water the Coulomb force dominates the dielectric term at frequencies below the charge relaxation frequency since the proportional variation in conductivity with temperature is greater than that of the permittivity. From these and other experiments [4, 21], velocities greater than $100 \mu \mathrm{m} \mathrm{s}^{-1}$ are easily achievable in the lower frequency range $(<200 \mathrm{kHz})$, the optimal range for REP.

This fluid motion is not natural convection. Previous experiments have shown that, with $1064 \mathrm{~nm}$ highly 

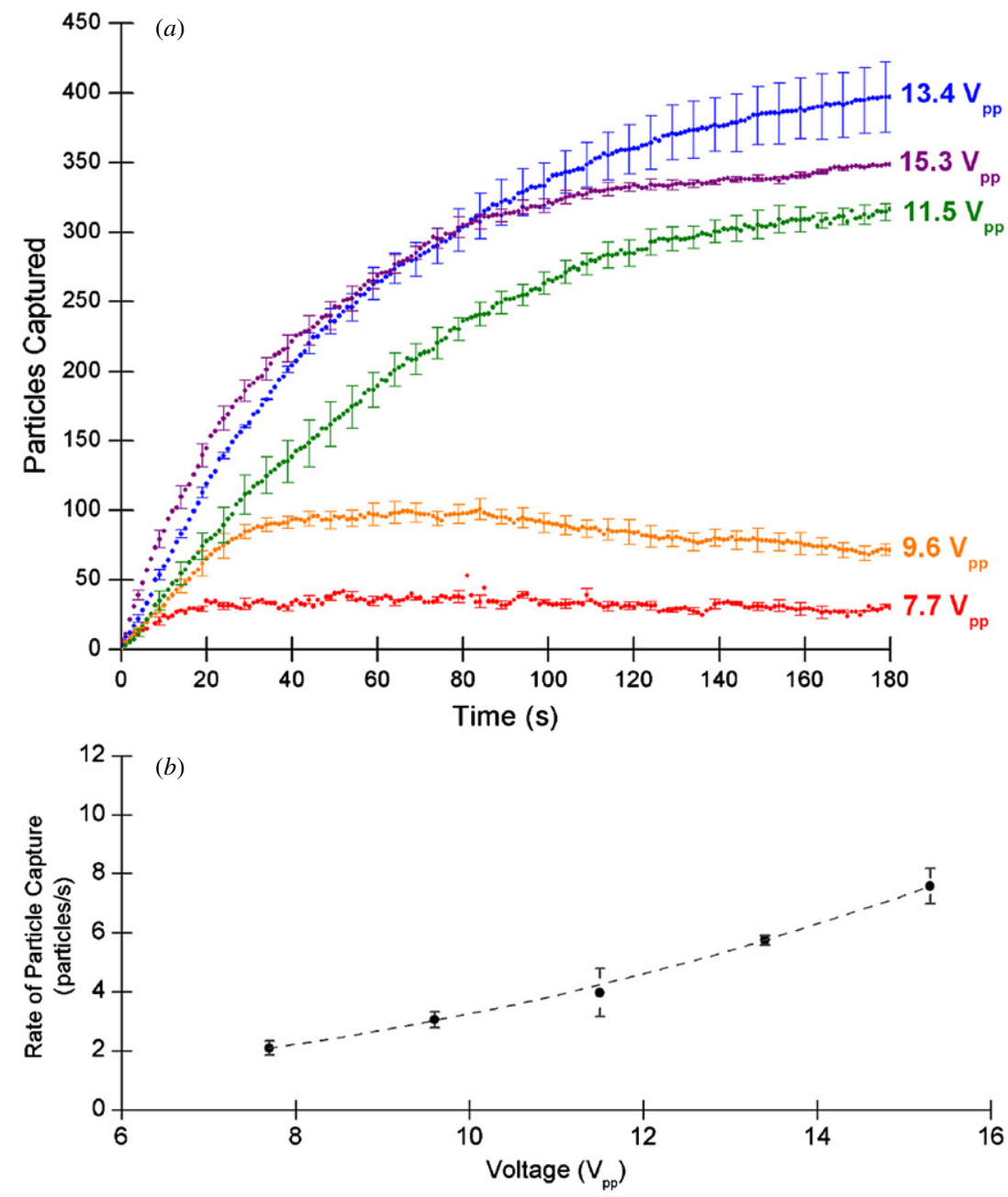

Figure 4. (a) The accumulation of $1.0 \mu \mathrm{m}$ particles on the surface of an ITO electrode over a period of 3 min. Five separate voltages were applied while illumination intensity $(23 \mathrm{~mW})$ and ac frequency $(70 \mathrm{kHz})$ were held constant. $(b)$ The rate of particle capture for the first $10 \mathrm{~s}$ after actuation of REP. The dashed line is a second-order polynomial fit and error bars represent one standard deviation.

focused optical traps in water, the temperature increase is approximately $1.4{ }^{\circ} \mathrm{C}-1.9{ }^{\circ} \mathrm{C}$ per $100 \mathrm{~mW}$ [53]-the typical illumination used for these REP experiments was approximately $20 \mathrm{~mW}$. A comparative analysis of different forces in microsystems shows that the buoyancy forces are usually unimportant when electrical forces are present [36]. Further, the microfluidic chamber height was $50 \mu \mathrm{m}$, and at heights less than the order of $1 \mathrm{~mm}$, gravity cannot overcome viscous friction [54]. Finally, experiments performed in the absence of the electric field demonstrated no observable fluid motion. REP therefore requires simultaneous application of the illumination and the electric field in order to concentrate particles.

\subsection{Characterization of colloidal accumulation}

REP of $1.0 \mu \mathrm{m}$ polystyrene particles as a function of voltage was conducted at $23 \mathrm{~mW}$ and $70 \mathrm{kHz}$. The results for five different applied voltages are shown in figure 4(a). In general, the rate of particle collection increases linearly $(<20 \mathrm{~s})$ and then the number of particles gradually plateaus, reaching a saturation point. The linear rate of particle capture was calculated for the first $10 \mathrm{~s}$ after actuation of the REP, and the results are shown in figure $4(b)$. The rate of particle capture gives insight into the physical behavior of REP and can be used to validate the proposed mechanisms driving REP. Similar analysis was applied to the frequency and illumination experiments to obtain the initial rate of particle capture.

The final number of captured particles increased as voltage increased, except for the highest applied voltage $\left(15.3 \mathrm{~V}_{\mathrm{pp}}\right)$. For this case, the particle-electrode holding force was unable to overcome hydrodynamic lifting forces and therefore not as many particles could be trapped. From equation (1), it is expected that the fluid velocity, and therefore the introduction of particles, increases with the square of the applied voltagefigure $4(b)$ confirms this trend with the experimental data points fit with a second-order polynomial.

Next, the frequency-dependent concentration of particles was investigated at $23 \mathrm{~mW}$ and $15.3 \mathrm{~V}_{\mathrm{pp}}$. For each trial, frequency was varied in steps of $15 \mathrm{kHz}$ from $25 \mathrm{kHz}$ to $100 \mathrm{kHz}$ with an additional data point at $110 \mathrm{kHz}$, as no particle trapping was observed at $115 \mathrm{kHz}$. The accumulation for each frequency over the collection period is shown in figure $5(a)$. The rate of particle capture as well as the number of particles captured after $3 \mathrm{~min}$ is shown in figure $5(b)$. 

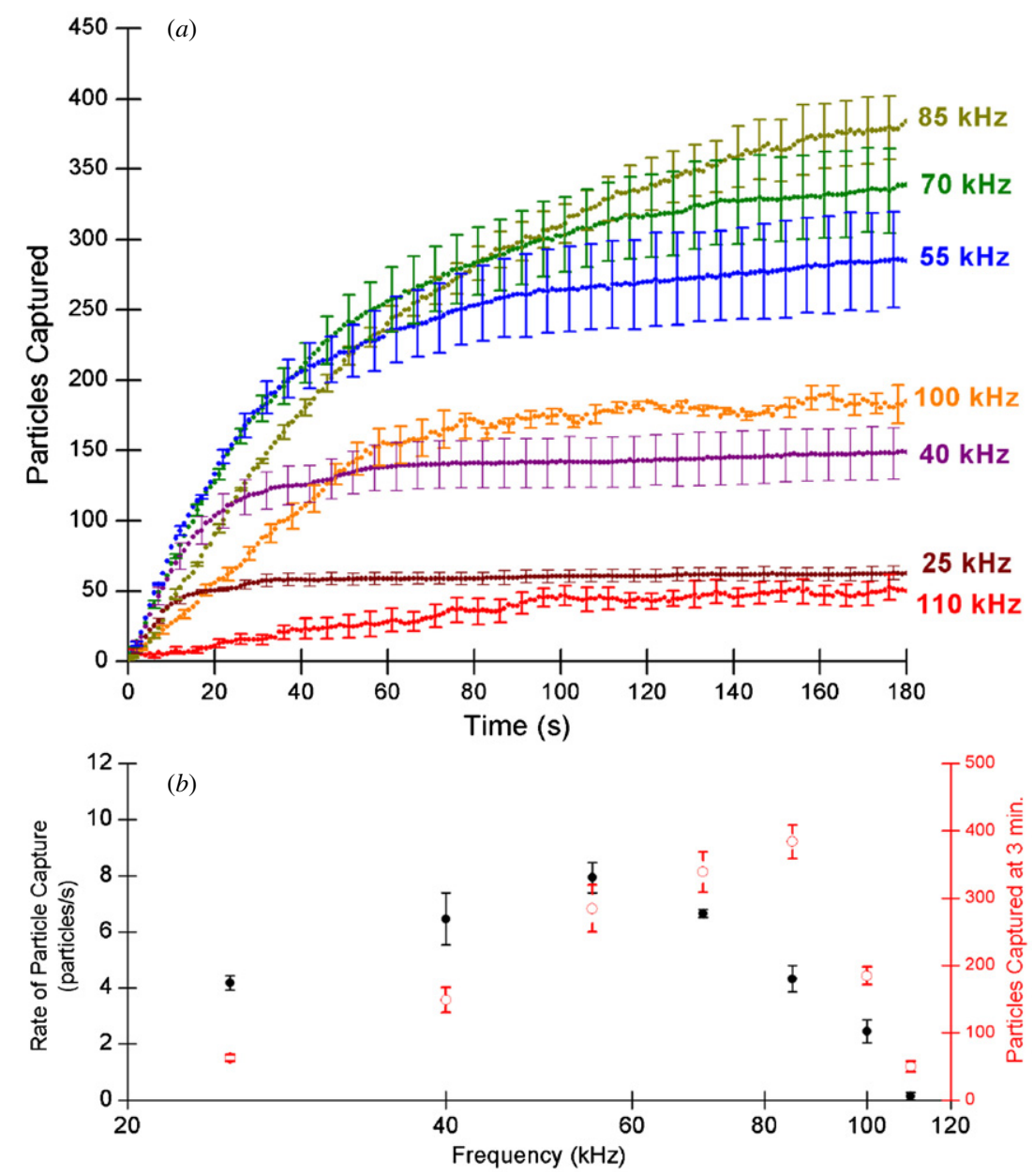

Figure 5. (a) The accumulation of $1.0 \mu \mathrm{m}$ particles on the surface of an ITO electrode for various frequencies, $15.3 \mathrm{~V}$ pp and $23 \mathrm{~mW}$. (b) The rate of particle capture $(\bullet)$ and number of captured particles at $3 \mathrm{~min}(\circ)$. Error bars represent one standard deviation.

The greatest number of captured particles after $3 \mathrm{~min}$ occurred for $85 \mathrm{kHz}$, whereas the greatest rate of particle capture occurred at $55 \mathrm{kHz}$. Electrode-particle forces are inversely proportional with frequency. As the applied frequency decreased, it was observed that particles would migrate toward each other and toward areas of non-uniform electric field features on the electrode surface (scratches, etc) outside of the REP trapping region. This unwanted particle motion would overcome fluid drag from the generated electrothermal microfluidic vortex, leading to lower particle aggregation and trapping rates as frequency decreased. The number of captured particles also decreased at higher frequencies $(>85 \mathrm{kHz})$ until, at $115 \mathrm{kHz}$, no particle trapping was observed even though particles were freely following the flow of the microfluidic vortex. The mechanism responsible for the particle-electrode trapping force is due to the polarization of the ionic double layer which will eventually disperse at higher frequencies. This relaxation was used to sort particles based on their polarization behavior in the following section.

To further characterize REP, the intensity of the 1064 $\mathrm{nm}$ illumination was varied $(23-86 \mathrm{~mW})$, while the ac signal remained constant at $70 \mathrm{kHz}$ and $15.3 \mathrm{~V}_{\mathrm{pp}}$ (figure 6). Measurements with a temperature-dependent fluorescent dye
(Rhodamine B) confirmed that both the maximum temperature and temperature gradients were proportional to the applied laser power (data not shown). Results shown in figure 6(b) exhibit a linear increase of the rate of particle capture with increased applied illumination-this response was expected as the velocity of the microfluidic vortex is proportional to temperature gradients $(\nabla T)$.

However, various effects at applied laser powers greater than $90 \mathrm{~mW}$ were observed including (i) bubble formation, (ii) permanent adhesion of particles to the electrode surface and (iii) trapped particles along the aggregation perimeter eventually being stripped away. This last effect occurred since the electrode-particle forces are independent of temperature gradients, and the larger imposed fluid velocities would produce hydrodynamic lift on the particles greater than the holding forces. Although these effects did not happen frequently, experimental results at these higher laser powers were inconsistent.

\subsection{Frequency-dependent electrokinetic separation of particles by type and size with REP}

The frequency-dependent polarization of the particles' ionic double layer responsible for inducing the electrokinetic forces for REP particle concentration can be utilized to separate 

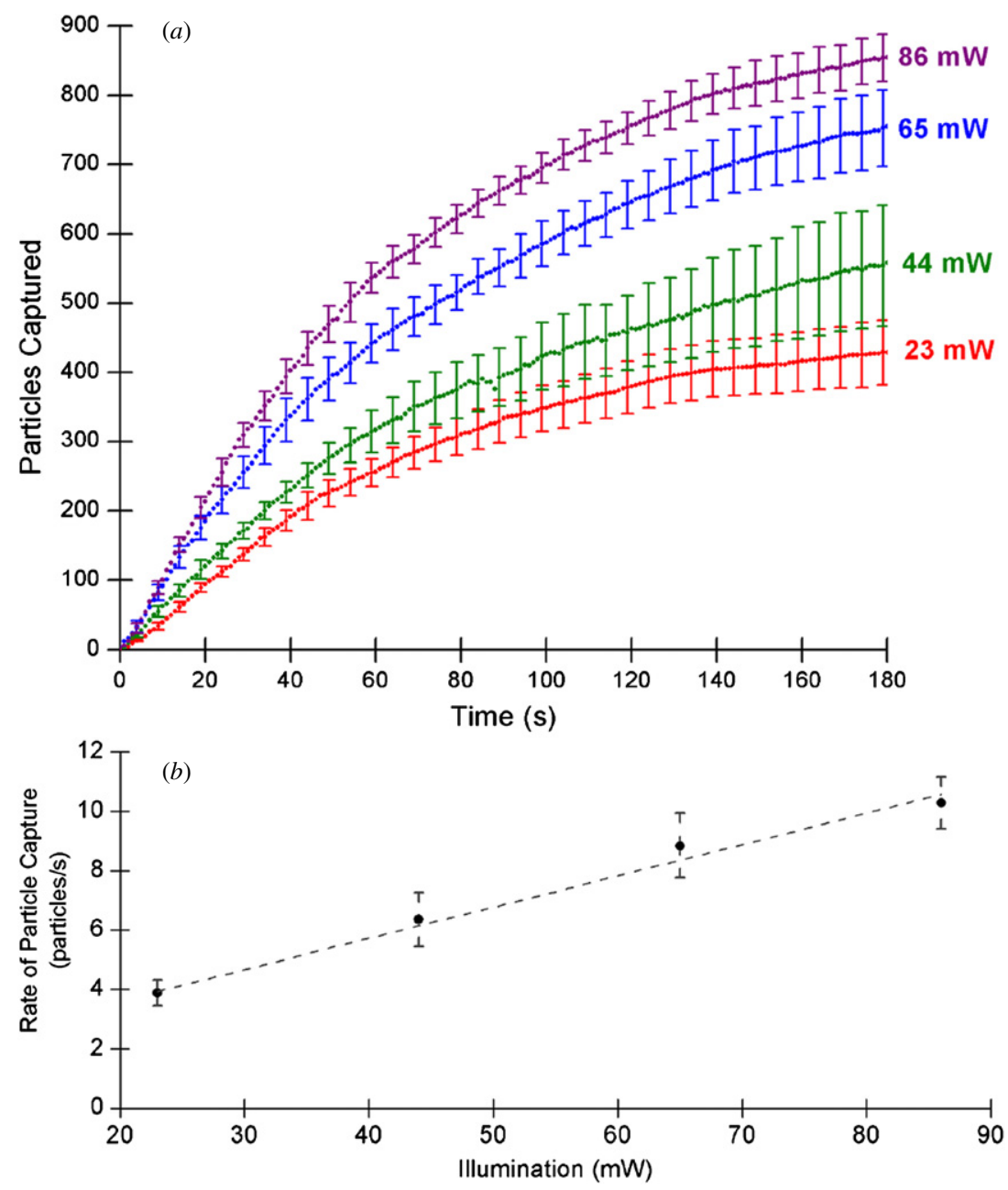

Figure 6. (a) The accumulation of $1.0 \mu \mathrm{m}$ particles for various illumination intensities while the applied voltage $\left(15.3 \mathrm{~V}_{\mathrm{pp}}\right)$ and ac frequency $(70 \mathrm{kHz})$ were held constant. $(b)$ The rate of particle capture for the first $10 \mathrm{~s}$ after actuation of REP. The dashed line represents a linear fit and error bars represent one standard deviation.

particles. A simple application to confirm the polarization behavior behind particle trapping is to sort similar particle types by size, as the relaxation frequency is inversely proportional to particle surface area $\left(a^{2}\right)$. An aggregation of polystyrene beads of three different sizes $(0.5 \mu \mathrm{m}$, $1.0 \mu \mathrm{m}$, and $2.0 \mu \mathrm{m}$ ) was captured at an initial frequency of $38 \mathrm{kHz}, 13.4 \mathrm{~V}_{\mathrm{pp}}$ and $23 \mathrm{~mW}$ (figure 7(a)). The frequency was then increased to $80 \mathrm{kHz}$, and the previously captured $2.0 \mu \mathrm{m}$ particles were carried away by the vortex while the $0.5 \mu \mathrm{m}$ and $1.0 \mu \mathrm{m}$ beads remained (figure $7(b)$ ). As the frequency was further increased to $106 \mathrm{kHz}$, the $1.0 \mu \mathrm{m}$ particles left (figure $7(c)$ ). Larger particles were being continuously carried into this region by the vortex but yet were not captured while the $0.5 \mu \mathrm{m}$ remained trapped. A video showing this sorting capability is available in the online version of the journal at stacks.iop.org/JMM/20/015022/mmedia (supplementary video 3).

The maximum frequency at which particles could be captured with REP was measured for each of the three particle sizes for a variety of applied potentials. The maximum REP trapping frequency is plotted versus particle diameter in figure $7(d)$. The fit (dashed line) is a second-order polynomial which is in agreement with theory. Therefore, REP can be used to trap and sort particles due to their frequency-dependent double-layer polarization behaviors.

To further exhibit this behavior, silica (non-fluorescent) and polystyrene (fluorescent) particles each $1.0 \mu \mathrm{m}$ in diameter were examined with REP. Under an initial condition of $23 \mathrm{~mW}, 150 \mathrm{kHz}$ and $19.8 \mathrm{~V}_{\mathrm{pp}}$ only polystyrene particles were captured. As the applied frequency decreased, more polystyrene particles aggregated until, at $130 \mathrm{kHz}$, the first captured silica particle was observed (figure 8(a)). More particles of both types were collected at lower frequencies, as shown in figure $8(b)$ at $90 \mathrm{kHz}$. For this example, the double-layer relaxation frequency for silica occurred at a lower frequency than polystyrene particles.

To further illustrate the separation of similar-sized particles, an additional fluid sample at $4.1 \mathrm{mS} \mathrm{m}^{-1}$ containing both $1.0 \mu \mathrm{m}$ silica and polystyrene particles was prepared. This sample was injected into the chamber with continuous, gravity-driven flow. With an applied signal of $200 \mathrm{kHz}$ and $14 \mathrm{~V}_{\mathrm{pp}}$, no particles were collected. At $120 \mathrm{kHz}$, only polystyrene particles were trapped on the electrode surface and this aggregation grew with the continuous introduction 

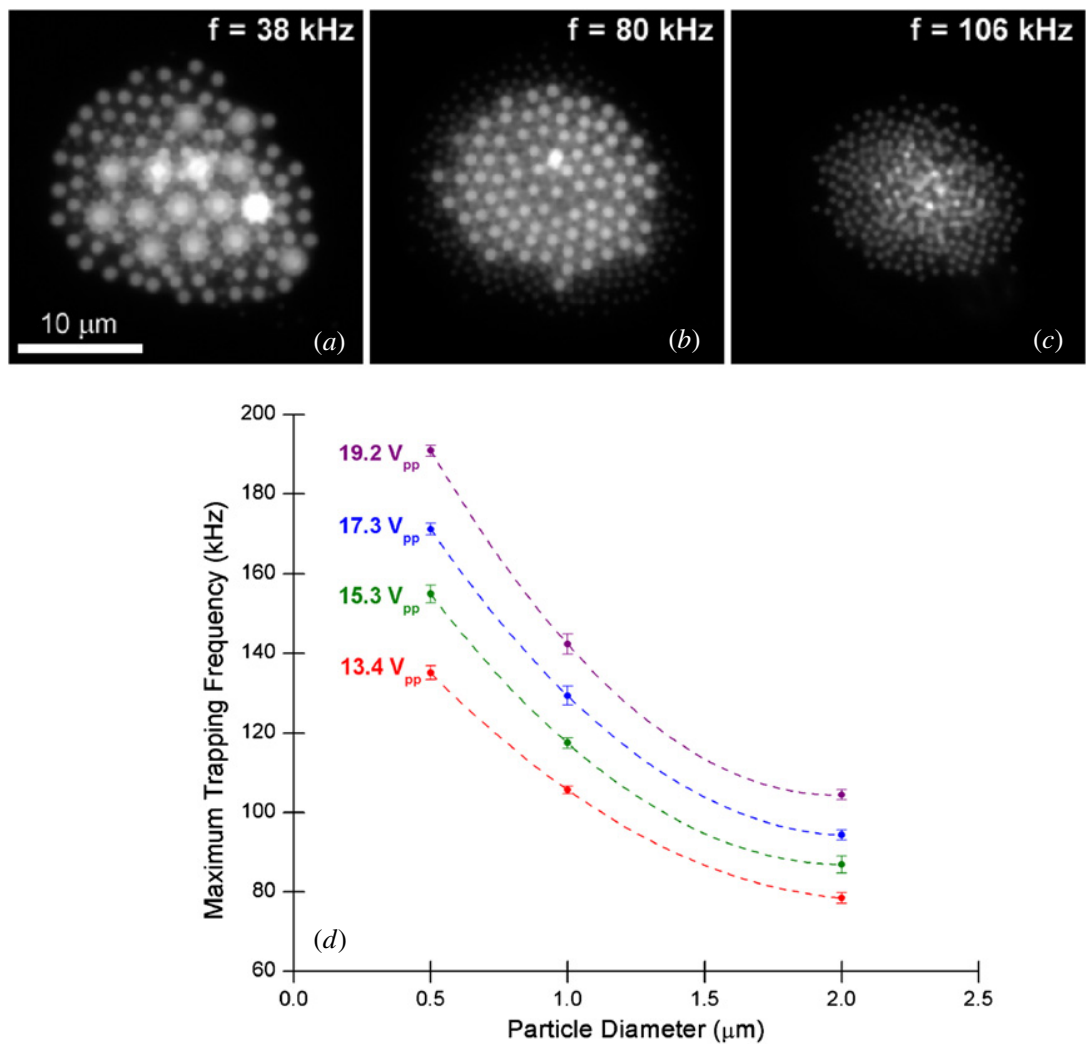

Figure 7. REP can be used to capture and separate like particles based on their size. (a) $2.0 \mu \mathrm{m}, 1.0 \mu \mathrm{m}$ and $0.5 \mu \mathrm{m}$ polystyrene particles were all initially captured at $38 \mathrm{kHz}$. (b) $2.0 \mu \mathrm{m}$ particles were carried away at $80 \mathrm{kHz}$. (c) Only $0.5 \mu \mathrm{m}$ particles remained at $106 \mathrm{kHz}$. (d) The maximum trapping frequency for these particles. The fit is a second-order polynomial, which is in agreement with theory. Error bars represent one standard deviation.

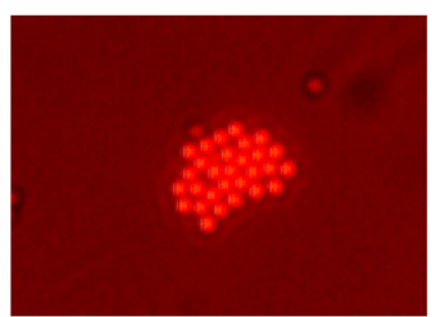

(a)

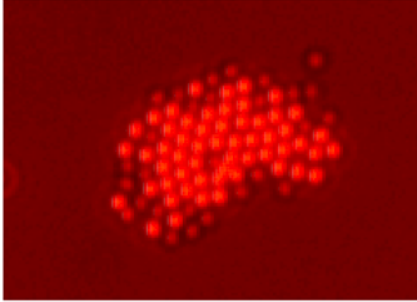

(b)

Figure 8. REP can be used to capture and sort unlike particles based on their polarization behavior. Silica (non-fluorescent) and polystyrene (fluorescent) particles each $1.0 \mu \mathrm{m}$ in diameter were used. (a) Polystyrene particles only were captured at $150 \mathrm{kHz}$ until, at $130 \mathrm{kHz}$, the first silica particle was trapped. (b) Both particles are trapped at $90 \mathrm{kHz}$. The applied voltage and illumination were $23 \mathrm{~mW}$ and $19.8 \mathrm{~V}_{\mathrm{pp}}$, respectively.

of particles from the bulk fluid flow. Decreasing the frequency to $80 \mathrm{kHz}$ captured both silica and polystyrene particles (refer to supplementary video 4 , available online at stacks.iop.org/JMM/20/015022/mmedia). All particles were removed from the surface at $200 \mathrm{kHz}$.

Future experiments will explore this frequency-dependent polarization mechanism, including characterizing the holding electrokinetic forces as a function of applied voltage and medium conductivity. Preliminary results have shown the

frequency-dependent sorting of particles with REP, and therefore this technique can be used to enhance a variety of diagnostic lab-on-a-chip applications.

\section{Conclusions}

We have introduced a novel optically induced method that couples electrothermal microfluidic motion with lowfrequency electrokinetic colloidal aggregation. The advantages of REP over existing particle handling techniques include the following.

- Optical methods allow dynamic control over the placement and translation of concentrated colloids.

- Enabling REP is simple, requiring a highly focused beam of light applied to the surface of a conducting, planar electrode within a parallel-plate configuration without the need for photosensitive materials.

- With the induced microfluidic vortex, colloids are rapidly and continuously concentrated.

- Colloidal concentration can occur anywhere on the surface of a planar electrode.

REP is not restricted to one particular illumination wavelength or electrode material; particles were accumulated on gold surfaces with $532 \mathrm{~nm}$ light and on both gold and ITO surfaces with $1064 \mathrm{~nm}$ illumination. Particle accumulation was characterized by varying the applied voltage, frequency 
and illumination intensity. In addition, REP can capture and sort different particles based on their double-layer polarization by varying the applied frequency.

This technique can be used to dynamically, rapidly and continuously concentrate, translate, sort and pattern colloids. In addition, by applying a short dc voltage, particles were permanently adhered to the electrode surface. This particle adhesion technique has been demonstrated previously $[1,25,26]$. REP can be applied for a variety of colloidal concentration applications, including creating artificial architectures for photonic crystals. Further, the frequency-dependent polarization of particles within a prepared sample can be investigated without the interference of non-uniform electrokinetic mechanisms such as DEP or ac electroosmosis. REP simultaneously induces fluid motion to expedite sample concentration, improving the efficiency of lab-on-a-chip sensors and processes. In general, REP is a versatile tool that can be applied to a variety of existing labon-a-chip techniques.

An extensive video showcasing this novel electrokinetic technique is available online at the 2009 Gallery of Fluid Motion [55].

\section{Acknowledgments}

We thank Dan Mueth, Joseph Plewa and Byeong-Seok Chae at Arryx, Inc. for technical advice and providing access to a $532 \mathrm{~nm} \mathrm{Nd:YAG} \mathrm{laser} \mathrm{based} \mathrm{Bioryx}{ }^{\circledR} 200$. We would like to acknowledge support from NSF Grant CMMI-0654031. AK acknowledges partial support from the Adelberg Fellowship, Purdue University. SJW acknowledges support under a National Science Foundation Graduate Research Fellowship and Purdue University's Laura Winkelman Fellowship for Doctoral Studies in the School of Mechanical Engineering. We thank Dr Robert Keynton at the University of Louisville Center for Micro/Nano Technology and their staff, including Scott Cambron, for access to their facilities and services.

\section{References}

[1] Williams S J, Kumar A and Wereley S T 2008 Electrokinetic patterning of colloidal particles with optical landscapes Lab Chip 8 1879-82

[2] Williams S J, Kumar A and Wereley S T 2009 A simple, optically induced electrokinetic method to concentrate and pattern nanoparticles Nanoscale 1 133-7

[3] Kumar A, Williams S J and Wereley S T 2009 Optically modulated rapid electrokinetic patterning for micro and nano particles SPIE 7371737110

[4] Kumar A, Williams S J and Wereley S T 2009 Experiments on opto-electrically generated microfluidic vortices Microfluid. Nanofluid. 6 637-46

[5] Noda S and Baba T 2003 Optoelectronic Industry and Technology Development Association (Japan) Roadmap on Photonic Crystals (Dordrecht/Boston: Kluwer)

[6] Lin R-Z et al 2006 Dielectrophoresis-based cell patterning for tissue engineering Biotechnol. J. 1949-57

[7] Kellar K L et al 2001 Multiplexed fluorescent bead-based immunoassays for quantitation of human cytokines in serum and culture supernatants Cytometry 45 27-36

[8] Ashkin A 1970 Acceleration and trapping of particles by radiation pressure Phys. Rev. Lett. 24 156-159
[9] Ashkin A, Dziedzic J M and Yamane T 1987 Optical trapping and manipulation of single cells using infrared-laser beams Nature 330 769-71

[10] Curtis J E, Koss B A and Grier D G 2002 Dynamic holographic optical tweezers Opt. Commun. 207 169-75

[11] Grier D G 2003 A revolution in optical manipulation Nature 424 810-6

[12] MacDonald M P, Spalding G C and Dholakia K 2003 Microfluidic sorting in an optical lattice Nature 426 421-4

[13] Russel W B, Saville D A and Schowalter W R 1989 Colloidal Dispersions (Cambridge/New York: Cambridge University Press)

[14] Lyklema J et al 1991 Fundamentals of Interface and Colloid Science (London/San Diego: Academic)

[15] Kremser L, Blaas D and Kenndler E 2004 Capillary electrophoresis of biological particles: viruses, bacteria, and eukaryotic cells Electrophoresis 25 2282-91

[16] Pohl H A 1978 Dielectrophoresis: The Behavior of Neutral Matter in Nonuniform Electric Fields (Cambridge Monographs on Physics) (Cambridge/New York: Cambridge University Press)

[17] Jones T B 1995 Electromechanics of Particles (Cambridge/New York: Cambridge University Press)

[18] Morgan H and Green N G 2003 AC Electrokinetics: Colloids and Nanoparticles (Microtechnologies and Microsystems Series 2) (Philadelphia, PA: Research Studies Press)

[19] Chiou P Y, Ohta A T and Wu M C 2005 Massively parallel manipulation of single cells and microparticles using optical images Nature 436 370-2

[20] Hwang H et al 2008 Reduction of nonspecific surface-particle interactions in optoelectronic tweezers Appl. Phys. Lett. 92

[21] Mizuno A et al 1995 Liquid microvortex generated around a laser focal point in an intense high-frequency electric-field IEEE Trans. Ind. Appl. 31 464-8

[22] Green N G et al 2000 Electric field induced fluid flow on microelectrodes: the effect of illumination J. Phys. D: Appl. Phys. 33 L13-7

[23] Green N G et al 2001 Electrothermally induced fluid flow on microelectrodes J. Electrost. 53 71-87

[24] Nakano M et al 2007 Development of an optoelectrostatic micropump using a focused laser beam in a high-frequency electric field IEEE Trans. Ind. Appl. 43 232-7

[25] Trau M, Saville D A and Aksay I A 1996 Field-induced layering of colloidal crystals Science 272 706-9

[26] Trau M, Saville D A and Aksay I A 1997 Assembly of colloidal crystals at electrode interfaces Langmuir 13 6375-81

[27] Yeh S R, Seul M and Shraiman B I 1997 Assembly of ordered colloidal aggregates by electric-field-induced fluid flow Nature 386 57-9

[28] Ristenpart W D, Aksay I A and Saville D A 2004 Assembly of colloidal aggregates by electrohydrodynamic flow: kinetic experiments and scaling analysis Phys. Rev. E 69

[29] Hayward R C, Saville D A and Aksay I A 2000 Electrophoretic assembly of colloidal crystals with optically tunable micropatterns Nature 404 56-9

[30] Duhr S and Braun D 2005 Two-dimensional colloidal crystals formed by thermophoresis and convection Appl. Phys. Lett. 86

[31] Chiou P Y et al 2008 Light-actuated ac electroosmosis for nanoparticle manipulation J. Microelectromech. Syst. 17 525-31

[32] Hwang H and Park J K 2009 Rapid and selective concentration of microparticles in an optoelectrofluidic platform Lab Chip 9 199-206

[33] Valley J K et al 2008 Operational regimes and physics present in optoelectronic tweezers J. Microelectromech. Syst. 17 342-50 
[34] Choi W et al 2006 Lab-on-a-display: microparticles manipulation using liquid crystal display Int. Conf. on Microtechnologies in Medicine and Biology (Okinawa, Japan)

[35] Smith S P et al 1999 Inexpensive optical tweezers for undergraduate laboratories Am. J. Phys. 67 26-35

[36] Ramos A et al 1998 AC electrokinetics: a review of forces in microelectrode structures J. Phys. D: Appl. Phys. 31 2338-53

[37] Cherukat P and McLaughlin J B 1994 The intertial lift on a rigid sphere in a linear shear-flow field near a flat wall J. Fluid Mech. 263 1-18

[38] Fagan J A, Sides P J and Prieve D C 2004 Vertical motion of a charged colloidal particle near an AC polarized electrode with a nonuniform potential distribution: theory and experimental evidence Langmuir 20 4823-34

[39] Fagan J A, Sides P J and Prieve D C 2005 Evidence of multiple electrohydrodynamic forces acting on a colloidal particle near an electrode due to an alternating current electric field Langmuir 21 1784-94

[40] Fagan J A, Sides P J and Prieve D C 2002 Vertical oscillatory motion of a single colloidal particle adjacent to an electrode in an ac electric field Langmuir 18 7810-20

[41] Green N G and Morgan H 1999 Dielectrophoresis of submicrometer latex spheres: 1. Experimental results J. Phys. Chem. B 103 41-50

[42] Schwan H P et al 1962 On low-frequency dielectric dispersion of colloidal particles in electrolyte solution J. Phys. Chem. 662626

[43] Schwarz G 1962 A theory of low-frequency dielectric dispersion of colloidal particles in electrolyte solution J. Phys. Chem. 662636

[44] Hoggard J D, Sides P J and Prieve D C 2008 Electrolyte-dependent multiparticle motion near electrodes in oscillating electric fields Langmuir 24 2977-82
[45] Liu Y, Xie R G and Liu X Y 2007 Fine tuning of equilibrium distance of two-dimensional colloidal assembly under an alternating electric field Appl. Phys. Lett. 91

[46] Nadal F et al 2002 Electrically induced interactions between colloidal particles in the vicinity of a conducting plane Phys. Rev. E 658

[47] Santana-Solano J, Wu D T and Marr D W M 2006 Direct measurement of colloidal particle rotation and field dependence in alternating current electrohydrodynamic flows Langmuir 22 5932-6

[48] Crocker J C and Grier D G 1996 Methods of digital video microscopy for colloidal studies J. Colloid Interface Sci. 179 298-310

[49] Weber M J 2003 Handbook of Optical Materials (The CRC Press Laser and Optical Science and Technology Series) (Boca Raton, FL: CRC Press)

[50] Cheng J Y et al 2007 ITO patterning by a low power Q-switched green laser and its use in the fabrication of a transparent flow meter J. Micromech. Microeng. 17 2316-23

[51] Yavas O, Ochiai C and Takai M 1999 Substrate-assisted laser patterning of indium tin oxide thin films 5th Int. Conf. Laser Ablation COLA'99 (Gottingen, Germany)

[52] Ichikawa A, Arai F and Fukuda T 2006 Optical temperature control of multiple microheaters using digital micromirror device 19th IEEE Int. Conf. Micro Electro Mechanical Systems (MEMS 2006) (Istanbul, Turkey)

[53] Mao H B et al 2005 Temperature control methods in a laser tweezers system Biophys. J. 89 1308-16

[54] Gonzalez A et al 2006 Electrothermal flows generated by alternating and rotating electric fields in microsystems J. Fluid Mech. 564 415-33

[55] Williams S J, Kumar A and Wereley S T Optically induced electrokinetic patterning and manipulation of particles APS DFD Gallery of Fluid Motion [Video] 2008 Available at: http://hdl.handle.net/1813/11399 